PVP2006-93237

\title{
ASSET MANAGEMENT EVALUATION : THE KEY ROLE OF EXPERT ELICITATION
}

\author{
C. E. BAUBY \\ EDF - R\&D
}

6, quai Watier

78400 - Chatou - France

Phone / Fax : 33.1.30.87.79.39 / 82.13

Email : catherine.bauby@edf.fr

\author{
B. RICARD \\ EDF - R\&D \\ 6, quai Watier \\ 78400 - Chatou - France
}

\author{
P. HAIK \\ EDF - R\&D \\ 6, quai Watier \\ 78400 - Chatou - France
}

\begin{abstract}
The life management of a nuclear power plant raises several major issues amongst which ranks the aging management of the key components of the plant, both from a technical and an economic point of view. Decision-makers are thus faced with the need to define the best strategy in order to achieve the best possible performance which requires both a very precise modeling of the plant and a detailed analysis of all risks potentially incurred.

In this paper, we wish to provide the reader with an overview of how advanced expert elicitation techniques can help identify, structure, quantify and feed sensitive data into a risk-based information system which can then be used for risk-based asset management evaluation.

First we focus on the way knowledge management techniques allow EDF to structure and collect life-cycle management data into knowledge-based information systems. The elicitation of component experts is key, particularly in order to get technical information on the future behavior of the component ("anticipation" of whatever events may occur on the plant).

We then detail how expert elicitations allow to quantify the probabilities of occurrence of the events: whether there is feedback data, models or not, expert opinion has to be taken into account and mixed, for instance with Bayesian procedures, to this information.

Lastly we describe how the information elicited from experts can help top level decision makers get a transverse, long term view on how life management investment strategy translates into plant availability, avoided costs and improved component durability.
\end{abstract}

\section{INTRODUCTION}

The management of a production asset, especially with a long-term goal to achieving an optimum lifetime, raises several major issues, among which rank the technical management of the plant, its economics and the fleet level perspective one has to adopt.

Decision-makers are therefore faced with the need to define long-term policies (until the end of the life of the asset), which take into account multiple criteria including safety (which is paramount) and performance.

In this paper, we first remind the reader of the EDF three-level methodology for nuclear asset management, as introduced in PVP 2003 [1] and PVP 2004 [2]. The knowledge management techniques used to support the expert elicitation process (in order to anticipate whatever could occur to the plant) and which facilitate the structuring and the processing of the acquired knowledge are then presented. Emphasis is given to the methodology developed for the quantification of the events (i.e. their probabilities of occurrence, their consequences in terms of availability) and of the mitigation actions (i.e. their cost, duration and impacts on events' occurrences). Lastly, we describe how the methodology, the information elicited from experts and their implementation in a software tool can be used in order to process technical and economic indicators obtained at the plant level as well as the conclusions top level decision makers can draw from them in order to evaluate and analyze long term asset management strategies.

\section{EDF THREE-LEVEL METHODOLOGY FOR ASSET MANAGEMENT}

The asset management methodology presented in PVP 2003 [1] proposes to evaluate the status of a plant through the indicators (the safety level, the power level, the 
availability) as well as through economic quantifiers (such as investment, operational and maintenance costs) computed from data related to the decisions made in order to achieve a designated lifetime (yearly investments - potentially constrained -, yearly income, NPV - Net Present Value - of the studied strategy).

The methodology is divided into three levels. Each level addresses a quite specific function:

- the component/technical level: how to safely operate daily and invest for the future,

- the plant level: how to translate technical decisions into plant-wide consequences including economic performance and vice versa,

- the fleet level: how to manage a large number of similar assets.

Theoretically, levels two and three can be inferred from component-level technical, economic and financial assessments (long-term equipment reliability, maintenance strategies,...) by a "rolling-up" process.

The aim is to translate component-level estimates into a plant-wide decision process while taking into account the various sources of uncertainty associated with this assessment, as shown in Figure 1.

At the component level, all the information, both economic and technical, needed to evaluate the plant for the considered lifetime is required. More precisely, as shown in Figure 2, for a given SSC (System, Structure or Component), information must be compiled in order to:

- identify the systems and components that are part of the SSC and their functions,

- evaluate the current level of aging of the SSC (as far as regulation criteria or operation history are concerned),

- anticipate the behavior of the SSC in the future through "scenarios": events that may occur, possible mitigation actions to prevent or diminish the negative consequences of those events as well as their consequences on the SSC and on the plant.

"Rolling-up" consists in aggregating the componentlevel scenarios for each SSC into a plant-wide scenario that takes into account the interactions existing between the components of the plant. Expected future values of the proposed indicators for this consolidated plant-level scenario can be computed. This can help decision-makers in evaluating a plant-level strategy.

\section{KNOWLEDGE MODEL AS A SUPPORT FOR EXPERT ELICITATION AND TECHNICAL AND ECONOMIC PLANT ASSESSMENT}

The evaluation of a production asset on a long-term basis requires all the information concerning what may occur on the asset, what decisions can be taken to manage the asset, and the potential consequences of those decisions and events on the asset.

This information is part of the knowledge of component experts and has not been fully formalized.

It is therefore necessary to identify the knowledge of component experts and structure it, so that it can be used by decision-makers to evaluate the plant according to the threelevel methodology for asset management.

In order to ensure consistency in the acquisition process and upholding of this knowledge, we identified the development of a knowledge model as a valuable prerequisite. This knowledge model defines formal interconnected structures that are used to classify, represent and utilize knowledge elements.

Such a model constitutes a common language between experts and decision-makers. It provides a stable structure that is then filled with appropriate information in order to produce relevant results. To make software design and usage more straightforward, the model also serves as a basis for the specification of data structure for dedicated tools to perform asset management analyses.

More precisely, the model can:

- sustain identification, acquisition, persistence of the relevant knowledge of component experts,

- enable sharing of this knowledge among experts when needed,

- make this knowledge available to decision-makers,

- be implemented into a tool to support decisionmakers' analyses.

For the considered domain of asset management evaluation of power plants, knowledge management techniques have been used and a model was developed to represent relevant knowledge. This model was presented in detail in PVP 2004 [2].

\section{SPECIFIC METHODOLOGY FOR EXPERT ELICITATION}

As highlighted by the pilot case study presented in PVP 2005 [4], the quality of the technical and economic results processed at the plant level highly depends on the quality, the homogeneity and the availability of the input data. As most of them must be elicited from the experts for each SSC, we focused our recent work on developing a more robust methodology - to support the expert elicitation process - and elaborating a set of dedicated tools.

\section{A dedicated methodology for risk anticipation, mitigation action identification and strategy elaboration}

Anticipating the future is a difficult exercise for component/material experts that are more familiar with data feedback analyses and extrapolation. Thus, it has been necessary to provide them with a methodology (and a set of tools) that can help them while trying to forecast what could happen to such or such SSC.

The methodology EDF designed relies on our previous work and operational experience in plant technical and economic assessment as well as on a 3-year PhD-thesis work that aimed at developing a methodology for components and systems aging anticipation based on experts crossed stimulation [3]. This latter work highlights the benefits of using creative techniques such as incremental filtering 
approaches in anticipation processes (cf. the Incremental Filtering Approach for Aging Anticipation shown in Figure 3). As shown in Figure 4, we decomposed the process of elaborating a SSC-level strategy (taking into account what its future might be) into two separated and sequential phases:

- A global and generic phase in which (for a given SSC, whatever the plant of the fleet considered) experts are asked to:

- anticipate any major risks (aging, obsolescence, regulatory constraints, ...) that may occur in the future and the associated events,

- identify any existing or to-be-developed mitigation actions that can be used to deal with these events.

- A specific phase in which (for a given SSC and a given plant of the fleet) experts are asked to:

- determine the relevant events amongst those identified during the generic phase,

- $\quad$ set up a strategy of mitigation actions to postpone the unwanted events or to mitigate their effects, in order to operate the component until a target year.

Each of these phases is then decomposed into steps in order to facilitate the elicitation process and to use the different tools that have been developed to support them.

Expert elicitation consists of operations aimed at identifying, gathering, structuring and saving relevant information held by component or material experts. It is performed by analysts, hereafter called "interviewers" to outline the fact that the main part of their work is based on preparing, conducting and analysing expert interviews. It appears that this elicitation task should be performed by dedicated analysts along with the experts themselves: firstly, it is not realistic to ask experts to master the knowledge model, representation techniques and elicitation methodology, secondly, an interviewer will have an external gaze which is helpful in avoiding implicit knowledge not be taken into the model, in ensuring consistency between experts, in "challenging" experts to avoid unnecessary complexity as well as oversimplification.

The EDF methodology provides a theoretical guideline that must be followed for expert elicitation in order to :

- identify, for each step, the input data on which they must rely and the output data expected,

- $\quad$ fill in the knowledge model that will finally be used to process the technical and economic results that allow strategy analyses,

- collect high quality homogenous data for all the studied SSC.

\section{EDF tools for risk anticipation, mitigation action identification and strategy elaboration}

In order to support the experts' elicitation done by interviewers, a set of two operational tools was developed :

- an operational guide for risk anticipation, mitigation action identification and strategy elaboration,
- $\quad$ an operational guide for events and mitigation actions quantification which proposes a criticity dependent strategy for the quantification of the probabilities of occurrence of the events (critical events must be estimated with precision as they will have much more impact on the processed technical and economic results).

The operational guide for risk anticipation, mitigation action identification and strategy elaboration includes:

- the identification of available and usable data (documents, projects, databases, etc.) or organisations,

- $\quad$ sets of questions and check-lists for each step of the elicitation process in order to feed the discussion with the experts,

- generic lists (degradation mechanisms, etc.) and typologies (typology of events and of mitigation actions),

- a set of synthetic table sheets in order to present/analyse the collected data,

- a criticity scale in order to organize events into a hierarchy and to focus the quantification efforts on the most critical events (this criticity scale is also used to homogenize the events considered for each SSC),

- a set of slideshows, documents, and other resources the interviewers can use to better communicate with experts.

\section{EDF criticity scale and the associated event hierarchy}

In a standard way, we define the criticity of an event by the product of its probability of occurrence and the gravity of its consequences:

$$
\text { Criticity }=\text { Probability } \times \text { Gravity }
$$

To do so, we introduce a qualitative four level probability scale and a qualitative three level gravity scale to characterize the events.

Our probablity scale is based on the following classes:

- highly probable for events that will almost certainly occur during the expected duration of operation ;

- probable for events that have about 1 chance in 2 to occur during the expected duration of operation ;

- possible for the events that have about 1 chance in 4 to occur during the expected duration of operation ;

- unlikely for the events that have a smaller chance to occur during the expected duration of operation.

The gravity scale is based on the consequences of an event (direct consequences on the production, consequences of the associated mitigation actions, etc.):

- very serious, irreversible if the event can lead to a long unavailability, to high costs, to the total destruction of a system/component included in the $\mathrm{SSC}$, or to the shutdown of the plant; 
- serious if the event can lead to a temporary unavailability, to substantial costs, or to the partial destruction of a system/component included in the SSC;

- minor if the event can lead to the shutdown of a minor system/component of the SSC, to a short unavailability, or to light maintenance action such as control or part replacement.

We also adopted a 3-level discrete criticity scale (as shown in Figure 5):

- highly critical events (C1) that are both highly probable and very serious;

- uncritical events (C3) that are both unlikely and minor;

- $\quad$ critical events (C2), which are defined as not being in the first two classes $\mathrm{C} 1$ and $\mathrm{C} 3$.

We used this criticity scale in order to organize events into a hierarchy both in a qualitative way (from expert judgement and before any further quantification) and in a quantitative way (after having quantified the probability of occurrence of the events and the consequences of the associated mitigation actions). This criticity scale is used as a filter to retain the most critical events and to focus our quantification work on the most critical events as it will be said in the following part.

\section{EDF tools to quantify the input data of the methodology}

Developed tools aim at supporting interviewers in the quantification of events and mitigation actions. For each event, a probability distribution of occurrence has to be defined ; for each mitigation action, its cost, impacts on the availability and power level of the plant, and its impacts on the probabilities of occurrence of events in the scenario must be precisely estimated in order to allow decision support evaluations, performed by the software tool.

Probability distributions of the events and effects of the mitigation actions on these distributions

Once the events and their possible associated mitigation actions have been determined, and if judged critical or highly critical with the criticity scale, their probability distributions must be precisely evaluated. Available information for this evaluation includes historical data (for instance observed times to failure if the event is related to the failure of an SSC), physical degradation/failure models, numerical codes or expert judgment if no more objective information is accessible. For each kind of information, specific probabilistic or statistical approaches are used to determine the appropriate probability distributions, as shown in Figure 6.

Let's focus on the case where no or too few objective information is available and subjective expert judgement is the only way to estimate the distribution of an event. For this precise case, a structured elicitation process has been developed. Here are its main steps :
- $\quad$ find the most appropriate experts to be interviewed (possibly a unique expert) ;

- write a document that describes the purpose and the objectives of the interview as well as the EDF asset management evaluation methodology and send it to the experts;

- $\quad$ select the appropriate interview mode in function of several criteria, such as for instance the expected number of elicited experts or their office location;

- run a preliminary session in which the interviewer will remind the experts of basic probabilistic notions and make them aware of classical biases involved in any elicitation process;

- ask precise questions on some characteristics of the probability distribution of the event (the values of some quantiles) in order to select the most appropriate family distribution and estimate the parameters of the distribution. A simple Excel tool has been developed to quickly draw the density function of the event from parameters given by the experts and thus make them graphically visualize the meaning of their answers and when necessary allow them to modify them;

- $\quad$ ask precise questions on how applying the different mitigation actions can modify the original probability distribution of the event;

- if necessary, combine results taken from different experts in order to obtain a single probability distribution for the event;

- $\quad$ send experts a report describing the results obtained.

\section{Other input data}

The other input data related to the SSCs, such as the costs of the mitigation actions of the events, the mean unavailability time due to the occurrences of the events, the impacts of the mitigation actions on the power level of the plant (increase or decrease) are given by the component expert(s), considering historical data and prospective estimations.

The data more related to the behaviour of the plant, such as the cost of one day of planned/unplanned unavailability and their expected evolution with time are given by the experts of the EDF economic division.

No particular interview process has been developed for these kinds of data, except the use of a systematic checklist in order to ensure to have all the data required to feed the knowledge database and the software tool that implements the methodology and performs actual studies.

\section{TECHNICAL AND ECONOMIC PLANT EVALUATION AS A SUPPORT FOR DECISION MAKERS}

Once the data needed have been elicited from the experts, they can be capitalized and processed in order to support decision makers in evaluating long-term strategies (comparison of alternative long-term strategies for a given SSC or a given pant, etc.). 


\section{Software tool}

A specific software tool was developed to implement the EDF three-level methodology for asset management and perform actual studies.

The tool is divided into 3 modules:

- a knowledge model management module: it is to be used by a knowledge-management specialist in order to implement the evolutions of the underlying model,

- a knowledge-base management module: component experts use it to fill in the information needed for asset management evaluation: events, probabilities of occurrence, mitigation actions, consequences on the components and on the plant... It also allows to overlay generic information with specific data to take into account the specifics of a given system,

- a scenario construction and evaluation module: the decision-maker uses it to describe studied scenarios and perform indicators evaluation.

The overall tool architecture and the mathematical techniques on which relies the scenario evaluation module are fully described in PVP 2005 [4].

\section{Computed indicators}

The indicators computed by the software tool are the following :

\begin{tabular}{|l|l|}
\hline $\begin{array}{l}\text { Cost of the } \\
\text { corrective } \\
\text { mitigation actions }\end{array}$ & $\begin{array}{l}\text { It is the sum of the costs of all corrective } \\
\text { mitigation actions taken in the "plant" } \\
\text { scenario }\end{array}$ \\
\hline $\begin{array}{l}\text { Cost of the } \\
\text { preventive } \\
\text { mitigation actions }\end{array}$ & $\begin{array}{l}\text { It is the sum of the costs of all } \\
\text { preventive mitigation actions taken in } \\
\text { the "plant" scenario }\end{array}$ \\
\hline $\begin{array}{l}\text { Cost of the planned } \\
\text { unavailability }\end{array}$ & $\begin{array}{l}\text { It is the cost that corresponds to the } \\
\text { planned unavailability due to } \\
\text { mitigations actions taken in the "plant" } \\
\text { scenario }\end{array}$ \\
\hline $\begin{array}{l}\text { Cost of the } \\
\text { unplanned } \\
\text { unavailability }\end{array}$ & $\begin{array}{l}\text { It is the cost that corresponds to the } \\
\text { unplanned unavailability due to the } \\
\text { occurrence of events and the associated } \\
\text { mitigation actions taken in the "plant" } \\
\text { scenario }\end{array}$ \\
\hline $\begin{array}{l}\text { Cost/Income due } \\
\text { to a change in } \\
\text { power level }\end{array}$ & $\begin{array}{l}\text { It is the cost or the income due to a } \\
\text { change in the power level of the plant } \\
\text { associated to the mitigations actions } \\
\text { taken in the "plant" scenario }\end{array}$ \\
\hline $\begin{array}{l}\text { Income from the } \\
\text { plant }\end{array}$ & $\begin{array}{l}\text { It is the income directly associated to } \\
\text { the operation of the plant }\end{array}$ \\
\hline NPV & Discounted Income minus Costs. \\
\hline
\end{tabular}

Example results can be found in the pilot case study presented in PVP 2005 [4]. The analyses of the results obtained allow decision makers to consider alternative strategies in order to optimize long-term plant operation and maintenance policies.

\section{Other useful functions}

As highlighted by the pilot case study introduced in PVP 2005, the results are sometime difficult to analyse. To facilitate the decision makers analyses we developed a few other functions :

- the most probable lifeline of the plant allows decision makers to identify what are the most probable events and mitigations actions for the studied plant

- a criticity-based filter allows the decision maker to rank events in terms of criticity according to the set of mitigation actions they have selected in the strategy they currently want to evaluate

- the lifeline finder allows the identification of events and mitigations actions whose conjunction leads to a given value of an indicator (mean, minimum, maximum, or any other encountered value, for a given indicator).

\section{CONCLUSION AND PERSPECTIVE}

The methodology and tools developed in order to facilitate the elicitation process - which is necessary to collect the input data needed by our evaluation tool - has been used this year, by a new team of interviewers to collect the data for our next pilot case studies.

A first analysis of the already collected data shows that the methodology and associated tools:

- facilitates expert elicitation process and interviewer training (as interviewers are not always experts in knowledge management or in probability assessment)

- reduces the interviewer dependency from collected data (as it leaves no place for interviewer subjective interpretations)

- is a guarantee of quality and homogeneity for collected data. The knowledge model used provides a consistent shell for all components, hence a standardization that is key for the "roll-up" step.

Used in conjunction with our 3-level methodology for asset management and the software tool that implements it, it can provide decision makers with high quality technical and economic evaluations that can help them in comparing alternative strategies for a given plant in order to optimize long-term plant operation and asset management strategies.

\section{REFERENCES}

[1] C. E. Bauby, V. E. Just, C. Garreau - Asset management evaluation methods: the EDF perspective - PVP 03 (20-24 July, Cleveland, USA).

[2] C. E. Bauby, P. Haïk, E. Remy, B. Ricard - Knowledge modelling for asset management evaluation - PVP 04 (25-29 July 2004, San Diego, USA).

[3] L. Bouzaiène, F. Pérès, P. Haïk - Maintenance Experts Crossed Stimulation: Application to the French Nuclear Fleet. ESREL 02, Lambda Mu 13 (19-21 March 2002, Lyon, France).

[4] C. E. Bauby, B. Charbonnier, P. Haïk, S. Lacombe, J. Lonchampt, E. Remy - Asset management evaluation : a pilot case study - PVP 05 (17-21 July 2005, Denver, USA). 


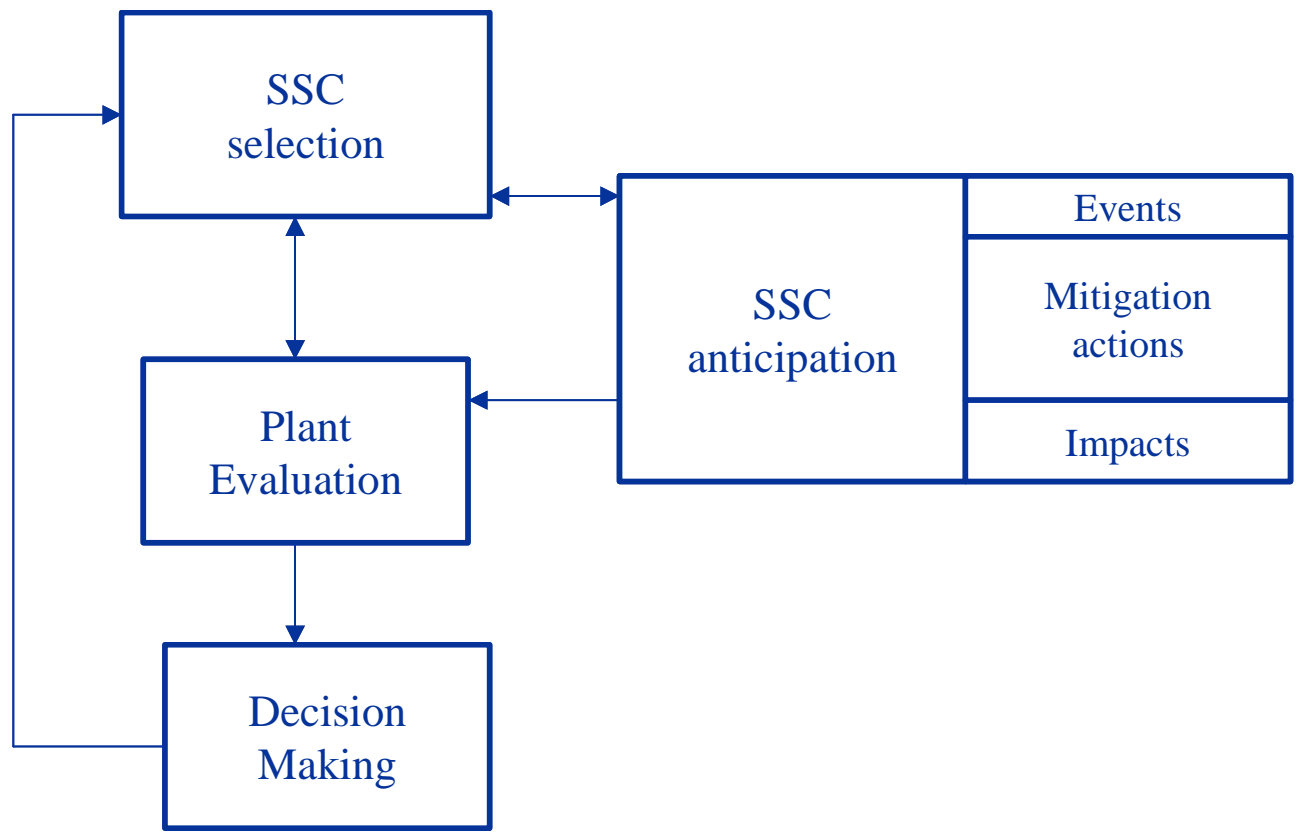

Fig. 1 - SSC management must be « rolled-up » to implement a plant-wide strategy

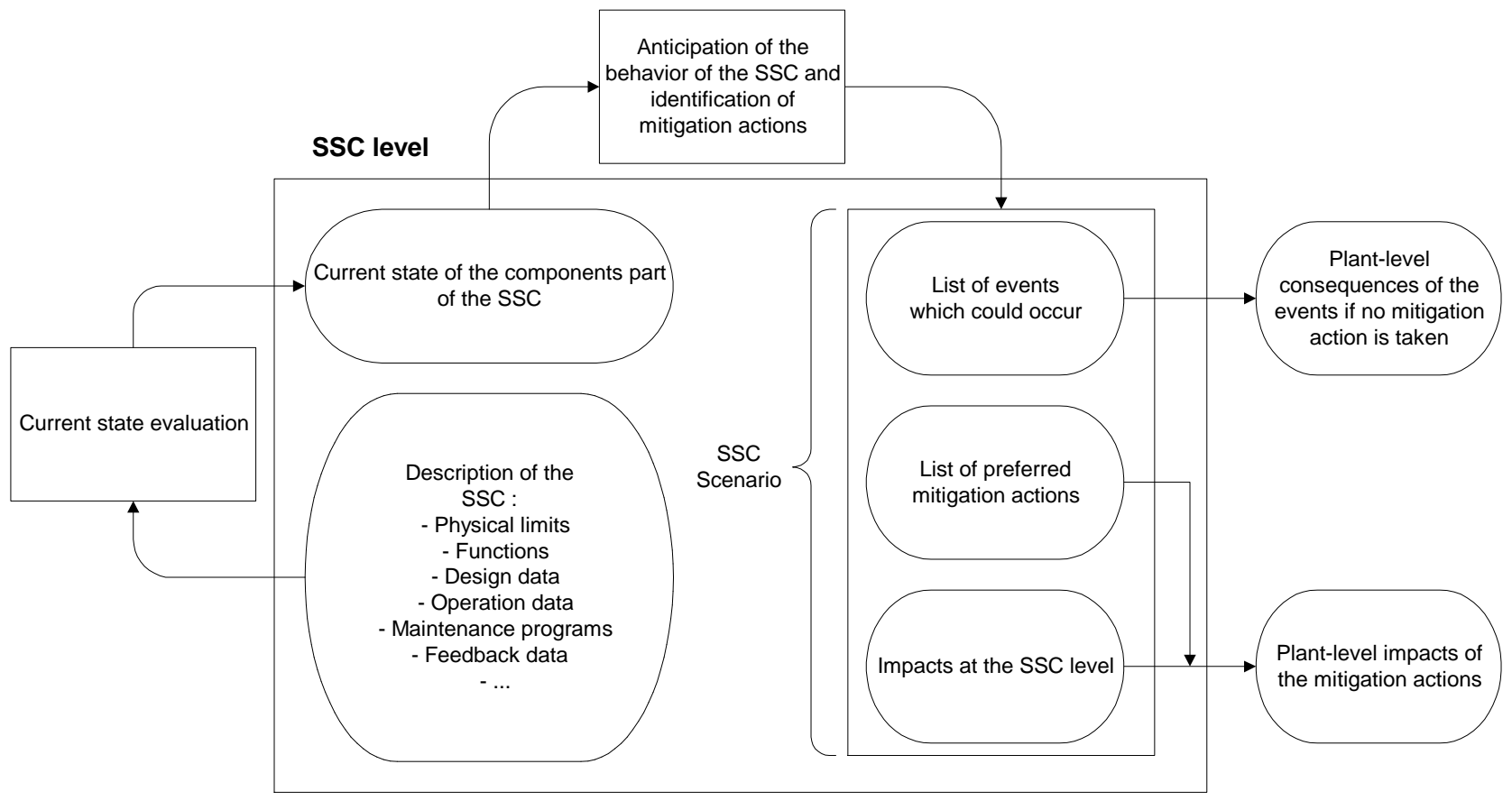

Fig. 2 - The key phases of SSC level management 


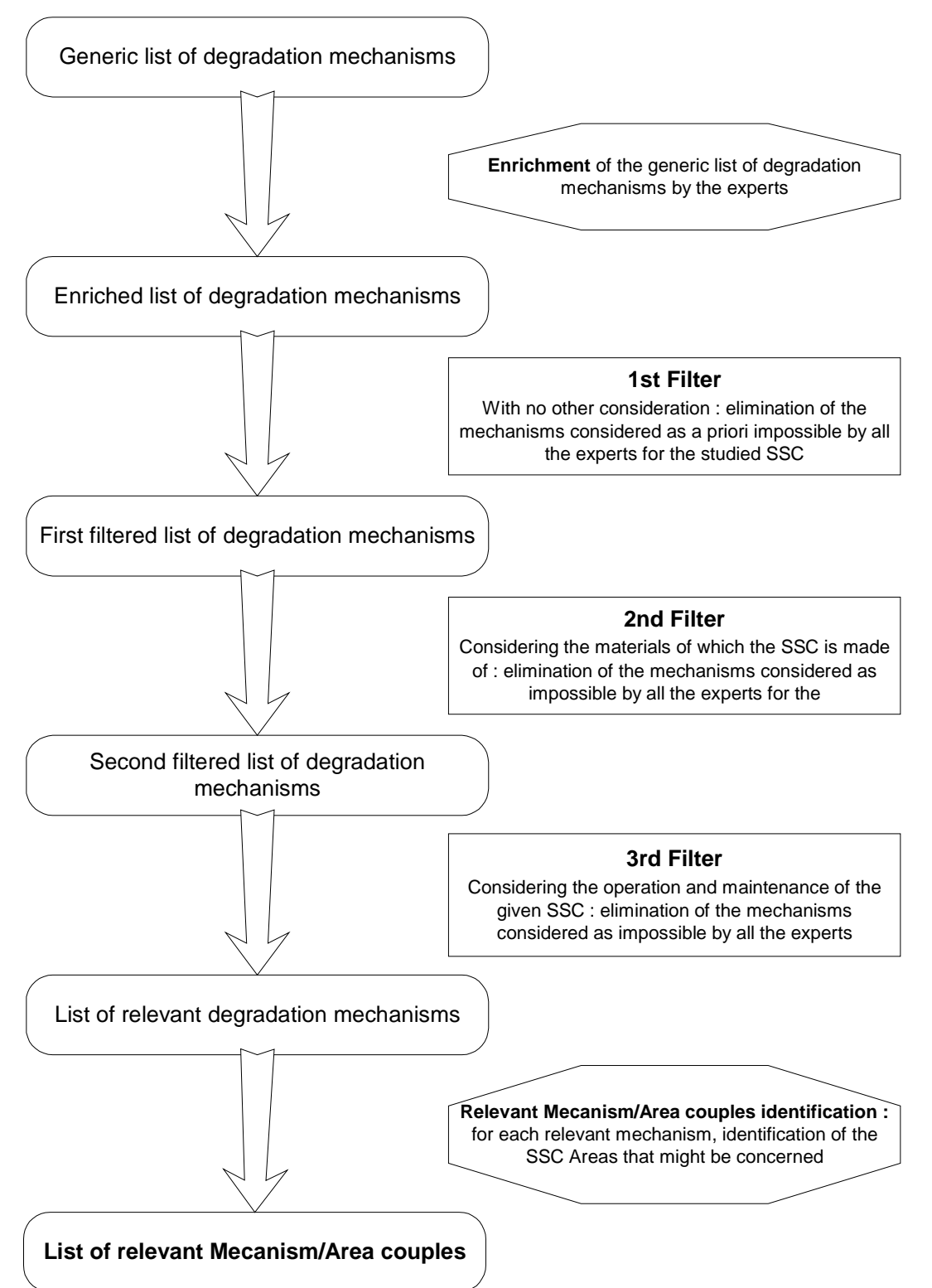

Fig. 3 - An Incremental Filtering Approach for Aging Anticipation 


\section{SSC-File Elaboration}

A fleet-wide risk analysis for a given SSC

\section{Scenario}

\section{Elaboration}

A plant specific risk anticipation, mitigation action identification \& strategy elaboration for a given SSC

\section{SSC Experts \& Existing Data,Identification}

\section{SSC's Definition}

- Material Decomposition of the Component(s)/System(s) included in

the SSC's scope

- Functional Decomposition of the SSC

\section{SSC's Current State evaluation}

- Design, Operation \& Maintenance pieces of information for the given SSC

- Regulatory concerns relevant for the given SSC

- Historical data (observed failures)

\& Main Risks Identification

Aging mechanisms

- External constraints (regulations, ...)

- Obsolescence, ...

4. Events Identification (Anticipation)

5. Mitigation Actions Identification

6. SSC-File Technical and Strategic Validation

For a given plant,

for which a technical and economic evaluation is expected

\section{SSC Experts Identification}

2. Plant Specificity Identification

\section{Scenario Elaboration \\ - Identification of the relevant events \\ - Mitigation actions Strategy Elaboration \\ - Events/Options Specific Quantification}

4. Scenario Technical and Strategic Validation

5. SSC Modelisation \& Evaluation using the dedicated tools

Fig. 4 - EDF methodology for expert elicitation 


\begin{tabular}{|c|c|c|c|}
\hline Unlikely & C2 & $C 3$ & $C 3$ \\
\hline possible & C2 & C2 & $C 3$ \\
\hline probable & C1 & $C 2$ & $C 2$ \\
\hline $\begin{array}{c}\text { highly } \\
\text { probable }\end{array}$ & $C 1$ & $C 1$ & $C 2$ \\
\cline { 2 - 4 } & very serious & serious & minor \\
\cline { 2 - 4 }
\end{tabular}

Fig. 5 - EDF discrete 3-level criticity scale

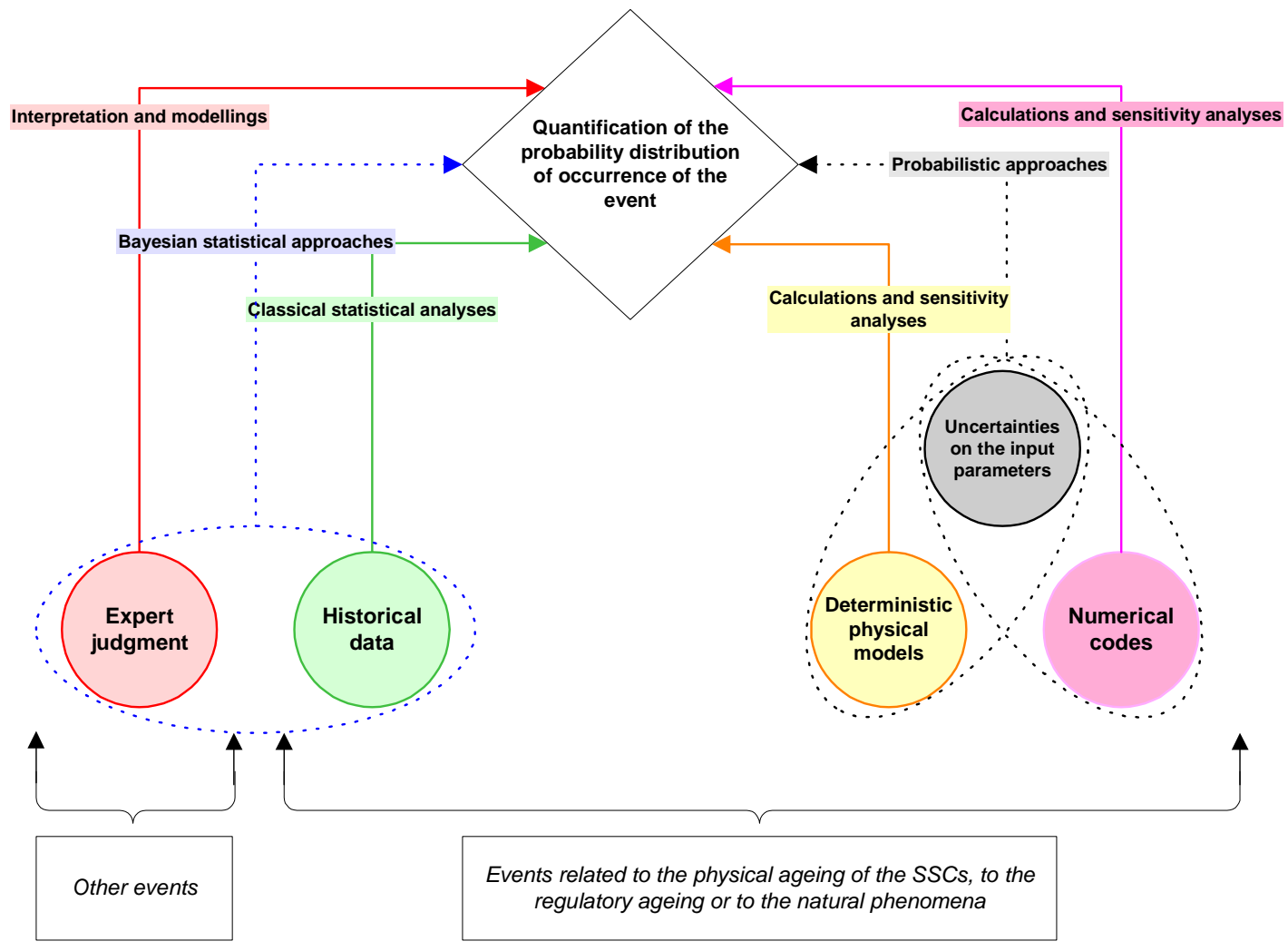

Fig. 6 - In function of the available information, the different possible approaches to evaluate the probability distributions of the events 